\title{
Effectiveness of an Secondary Prevention Program in Chronic Kidney Disease
}

\author{
Carlos Enrique Yepes Delgado*, Yanett Marcela Montoya Jaramillo, Beatriz Elena Orrego Orozco, \\ Paulina Bernal Ramírez, Luz Denise González, José Miguel Abad Echeverri, \\ María Patricia Arbeláez Montoya \\ University of Antioquia, Hospital Pablo Tobón Uribe, EPS SURA, Medellín, Colombia \\ Email: *caenyede@gmail.com
}

Received July 13, 2013; revised August 5, 2013; accepted August 14, 2013

Copyright (C) 2013 Carlos Enrique Yepes Delgado et al. This is an open access article distributed under the Creative Commons Attribution License, which permits unrestricted use, distribution, and reproduction in any medium, provided the original work is properly cited.

\begin{abstract}
Background: There are many programs which focus on late-stage chronic kidney disease (CKD), and it is considered that further evidence needs to be generated regarding the effectiveness of the programs used before renal replacement therapy. Study Design: A cohort study. Settings \& Participants: Patients over 15 years of age who had been diagnosed with CKD according to the KDOQI (Kidney Disease Outcomes Quality Initiative) guidelines and who had undergone conventional treatment (CT) or a renal protection program (RPP). These were patients of two Colombian health insurance companies. Predictors: Age, sex, marital status, comorbidities, CKD stage, and clinical indicators. Outcomes: First CKD progression, and need for renal replacement therapy (RRT). Measures: Clinical marker. Results: The RPP is structurally and functionally different from the CT. It offers the interdisciplinary management of patients, a greater number of medical appointments, and patients start to receive treatment at younger ages and at earlier stages of their condition. The clinical markers of the patients following the RPP are within adequate ranges, and their renal function is less impaired, despite the differences in basal conditions. Upon finishing the study, we found that patients who received CT had a higher risk of receiving nephrotoxic drugs and not receiving nephroprotective drugs. The explanatory variables for the first progression were age, stage, history of dyslipidemia, and hemoglobin, potassium, and albumin levels. These variables, together with glycemia levels were also valid for RRT, except for history of dyslipidemia, as it was not significant. Upon adjusting for the explanatory variables, it was found that belonging to the RPP and attending more appointments had a protective effect in the process of controlling renal damage. Limitations: A possible selection bias. Conclusions: Belonging to a structured renal protection program is an effective way to keeping the clinical markers associated with renal impairment within normal ranges.
\end{abstract}

Keywords: Chronic Kidney Disease; Renal Protection Program; Effectiveness; Clinical Markers; Progression of Renal Damage; Renal Replacement Program

\section{Introduction}

Due to the rapid increase in its prevalence, its close relationship with an increased risk of cardiovascular disease, and the high costs of treatment, chronic kidney disease (CKD) is now recognized as a public health problem that affects people of all ages and alters their quality of life $[1,2]$. Because of these characteristics, this disease is becoming a priority for healthcare systems. This has created an urgent need to develop effective ways of measuring interventions to prevent its progress $[3,4]$.

${ }^{*}$ Corresponding author.
According to the calculations of the National Health and Nutrition Examination Survey (NHANES) III, it is estimated that twenty million adults have CKD in the United States. Currently, approximately 360,000 patients have undergone renal replacement therapy (RRT) in the European Union. In developed countries, it is estimated that the number of people with end-stage CKD will continue to increase at an annual rate of around $5 \%$ to $8 \%$. This growth is driven by population aging and the increased incidence of diseases related to renal failure [5].

In Colombia, there are over 20,000 individuals with stage $5 \mathrm{CKD}$, with an age-adjusted prevalence of 454 
patients per million people. This figure is lower than the prevalence observed in the United States and higher than the average prevalence in Latin American countries in 2005. Nevertheless, the prevalence of early-stage CKD is only $0.87 \%$. This figure is lower than the one reported in other studies conducted on population samples and is thought to be the result of underreporting [6].

In order to delay CKD progression in Colombia, the Ministry of Social Protection implemented a model for its prevention and treatment in 2006. The model aimed to address the growing demand for services related to this condition [7,8]. Furthermore, Act 1122 of 2007 created the Cuenta de Alto Costo agency (CAC), a nongovernmental technical agency forming part of the Colombian General System for Health-Related Social Security. This is a strategy involving both public and private institutions and seeking to address the high costs generated by the condition itself, an issue that has a significant impact on Colombia [9]. However, before the appearance of such provisions, a Colombian health insurance company (EPS) designed and implemented a renal protection program (RPP) that was structurally and functionally capable of providing its users with a particular type of medical care that was very different from the conventional treatment $(\mathrm{CT})$ that other institutions continued to offer.

Although a number of national health institutions from across the world claim that it is important to reduce morbidity and mortality rates in the proactive management of CKD by making early referrals of patients to a multidisciplinary team, there are scant data within the current literature to support such a recommendation [10]. The authors of a meta-analysis published in April 2010 concerning evidence regarding the clinical and cost effectiveness of early referral strategies for managing people with markers of renal damage reported that, in the last two decades, only two studies in the world had focused on assessing the effectiveness of interventions carried out at early stages of CKD [11].

Not enough reports were found in the scientific literature that assessed RPPs which continuously monitored patients for at least four years and observed the relationship between the delay in kidney function impairment, and the behavior of variables such as clinical parameters (blood pressure, glycemia, lipid profile, etc.) and nutritional treatment [12-16].

Since the mechanisms for identifying CKD patients in the stages prior to RRT are not very effective, we are missing out many opportunities to implement strategies to reduce the progression speed of renal failure and thus reduce the incidence of terminal renal failure [6]. That is why this study aims to assess the effectiveness of the renal protection program in monitoring the clinical markers and their relationship with the progression of renal damage.

\section{Methods}

\subsection{Type of Study}

An analytic cohort study with ambispective follow-up given to two dynamic cohorts of patients over 15 years old and diagnosed with CKD. Individuals were classified by stage (according to the KDOQI guidelines) [17] in order to compare the behavior of the clinical and renal impairment indicators of the patients exposed to the RPP and those of the patients undergoing CT.

\subsection{Population}

The study was conducted with patients from two health insurance companies belonging to the same type of social security regime and operating in Antioquia, Colombia. Data was collected retrospectively from electronic medical records from April 1, 2004 (the starting date of the RPP) to the first half of 2007. After that date, information was collected prospectively until April 30, 2008. The total treatment time was 49 months. Figure 1 describes the participant selection process.

\subsection{Treatment Comparison}

The RPP is an interdisciplinary healthcare program based on a protocol involving periodic appointments and moni-toring of clinical and laboratory tests accompanied by continuous education. The RPP is aimed at CKD patients from the initial stages of their condition and it is divided into several levels. The first level is aimed at patients in stages 1 and 2. It offers medical appointments

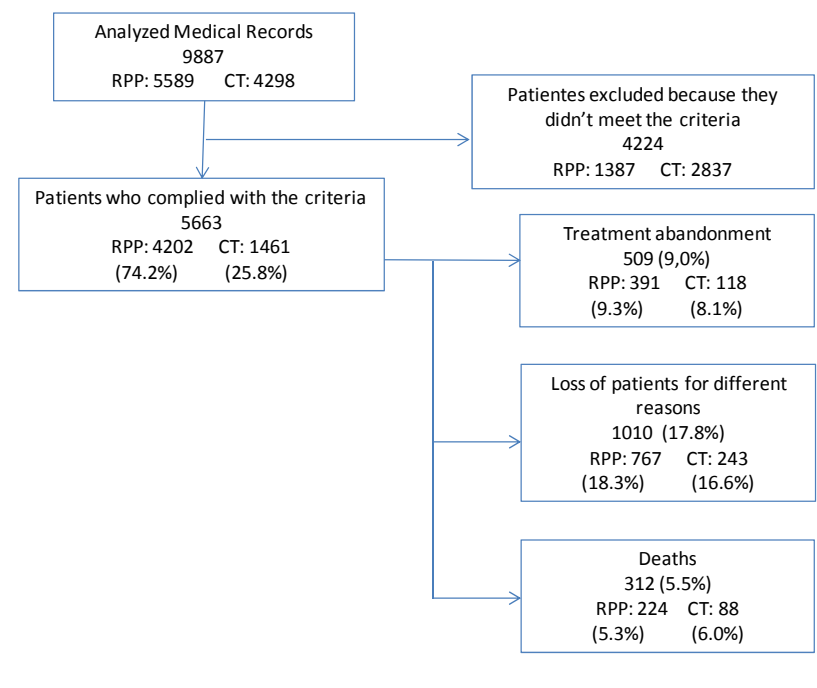

RPP: renal protection program. CT: conventional treatment

Figure 1. Flowchart depicting the CKD patient selection process in two Colombian private health insurance companies. Medellin 2004-2008. 
with interists and nutritionists on an annual and biannual basis, respectively. The second level is for patients in stages 3 and 4, and it offers medical appointments with internists, nephrologists, and nutritionists on a three-peryear and bimonthly basis, respectively. Laboratory tests are per-ormed one month prior to the medical appointment with a specialist.

When patients request a medical appointment because they feel the need to do it, conventional treatment (CT) either provides primary level attention or refers patients to a specialist, depending on the general practitioner's criteion.

No significant differences were found between the RPP and CT groups in terms of treatment abandonment, loss of patients for different reasons, and death $(\mathrm{p}=0.157 ; \mathrm{p}=$ $0.163 ; \mathrm{p}=0.318$ respectively).

\subsection{Variables}

The variables considered as criteria for CKD diagnosis were: creatinine clearance of less than $60 \mathrm{ml} / \mathrm{min}$, alrations observed in renal ultrasound tests, proteinuria levels higher than $150 \mathrm{mg} /$ day, alterations in urinary deposits, and -for diabetic patients- micro-albuminuria levels higher than $30 \mathrm{mg}$.

The variables included in the study were: age, sex, comorbidities, marital status, CKD stage according to the KDOQI guidelines, and clinical and laboratory variables as measured upon diagnosis and whenever stage progression took place. Some quantitative variables were categorized based on the clinical definitions for cutoff in accordance with the values reported in the literature

\subsection{Analysis Technique}

A comparison was carried out between the guidelines for clinical monitoring and care of patients with renal alterations used by the institution offering the RPP, and the treatment process used by the institution offering CT. The objective was to identify differences in the healthcare process, tests carried out, frequency of tests and appointments, types of professionals involved in the process, etc.

The population was characterized when CKD was diagnosed through frequency distributions and proportions for the qualitative variables. Quantitative variables were characterized via measures of dispersion and descriptive statistics. The Kolmogorov-Smirnov or Shapiro Wilk tests were also used. The qualitative variables of the two groups under study were then compared using the Pearson Chi2 test or the Chi2 test for trends, depending on the case. If the quantitative variables had a normal distribution, they were compared using the t-Student test. If the distribution was not normal, then the U Mann-Whitney test was used. The odds ratios (OR) and their corresponding confidence intervals were calculated to be $95 \%$.

A propensity score (PS) was used to assess effectiveness in maintaining the values for clinical markers and exposure to nephrotoxic and nephroprotective drugs within normal ranges. This statistical technique calculates the conditional probability of having been exposed to the variable of interest based on the subject's baseline features [18]. The PS is composed of the following variables: age $(>65$ years $/<=65$ years $)$, sex (female/male), marital status (no partner/ with permanent partner), arterial hypertension (yes/no), diabetes mellitus (yes/no) and dyslipidemia (yes/no). The PS created with these variables shows how they explain the probability of joining a RPP or undergoing CT by means of a logistic regression model that aims to predict exposure, and manages to group the various confounding variables into a single one [19]. These models also included the initial values of each variable.

Finally, two logistic regression models were created. Their dependent variables were the first progression into a later stage and the need for RRT. The variables included in the models were selected based on the Hosmer Lemeshow test. The quantity of data available for each variable was determined using the stepwise technique to obtain the final model. These models excluded patients who joined the study at stage 5 of their condition.

\subsection{Avoidance of Bias}

A potential bias could have arisen when we were forming the cohort and encountered participants with different features which increased the probability of having any of the outcomes. We therefore conducted multivariate analyses that adjusted for the features and basal conditions of the subjects. To avoid selection bias, we ensured that patients complied with the inclusion criteria. Possible migration bias could have occurred when the conditions of those who abandoned treatment were different from the conditions of those who stayed in it, and when such conditions were associated with the outcome itself. To prevent this, we inquired about censoring, either by reviewing the records of the health insurance companies or by asking via telephone. We thus located $80 \%$ of the patients and found no differences between the groups.

A possible reporting bias might have occurred when it was more likely to find more cases in the cohort being monitored for a longer time period (the RPP). Another bias resulted from the fact that the RPP actively searches for patients, thus its clinical records had more data than those of the patients following CT (underreporting). This bias was avoided by triangulating information and crossing the records for the missing data when possible. 


\section{Results}

When comparing the number of appointments attended by patients from both programs, it was observed that patients belonging to the RPP had more appointments with internists, as well as educational and nutritional appointments. The highest percentage of appointments for patients following CT was with general practitioners and ophthalmologists, as shown in Table 1.

There was a proportional difference in CKD stage at the moment of diagnosis between the patients from the two health insurance companies. More than $30 \%$ of the patients who joined the RPP were in the first two stages of the condition, compared to only $16.7 \%$ of patients who underwent $\mathrm{CT}$. In other words, CT had a higher number of patients in the final stages of CKD. Patients undergoing $\mathrm{CT}$ are more likely to be diagnosed with CKD when the condition is already in the final stages of its development, as shown by the OR represented in Table 2. According to this, a patient following $\mathrm{CT}$ is approximately $50 \%$ more likely to be diagnosed with late-stage CKD (stages 4 and 5) than a patient from the RPP.

Additionally, CKD patients following CT were significantly older (Me:70 years old) than RPP patients (Me: 66 years old). This was observed in both males and females.

Upon comparing the morbidities of the patients following CT and those of the RPP patients, it was observed that prevalence values were similar for both groups (Figure 2), except for arterial hypertension $(\mathrm{p}<0.001)$, dyslipidemia $(P=0.023)$, diabetes mellitus $(p=0.014)$, cerebrovascular disease $(0.002)$ and obesity $(p=0.017)$.

After analyzing the values observed for the various clinical markers at the moment of diagnosis, we found significant differences between the CT and RPP patients regarding serum creatinine, creatinine clearance, triglycerides, and potassium. These values were less affected or even within normal ranges for the RPP patients. The values for the high- and low-density lipoproteins were more favorable for the patients undergoing CT. No significant differences were found between the values for systolic arterial pressure, glycated hemoglobin, 24-hour proteinuria, total cholesterol, hemoglobin, phosphorus, and parathyroid hormone.

Likewise, upon analyzing the values at the moment of diagnosis, we found that the risk of receiving nephrotoxic drugs such as non-steroidal anti-inflammatory drugs or aminoglycoside antibiotics is 1.7 times greater for patients following CT (CI95\%: 1.4 - 2.1) than for patients in the RPP. We also found that the risk of not receiving nephroprotective drugs is 2.1 times higher (CI95\%: 1.8 2.4) for patients with CT than for those following a RPP.

The risk of having out-of-range values for the clinical markers and for the variable "final exposure to nephrotoxic and nephroprotective drugs" at the end of the treatment period was analyzed (Table 3). It was then found that CT patients had a higher risk of having clinical markers which were out of range for SAP, fasting blood glucose, glycated hemoglobin, and potassium. The risk of having out-of-range clinical markers for the cholesterol variable was higher in the RPP patients. No differences were found in albumin, calcium, and hemoglobin. Similarly, the risk of receiving nephrotoxic drugs for patients undergoing $\mathrm{CT}$ was 0.63 times greater than that of the patients in the RPP. The risk of not receiving nephroprotective drugs was 0.35 times higher for CT patients.

The models described below show the effect of the "first progression" and "need for RRT" variables on renal function impairment. The variables are adjusted for patient features (Table 4).

Regarding the variable representing the first progresssion into the next stage of CKD, we found that the patients in the RPP and those who attended more than eight medical appointments had a lower risk of CKD progresssion. Their protective fraction was $51.7 \%$ and $71.3 \%$ respectively. Likewise, a history of dyslipidemia, together with out-of-range levels of hemoglobin, potassium, albumin, and glycemia, behaves as a risk factor for CKD progression. On the other hand, out-of-range hemoglobin and albumin levels represent the highest risk (2.91 and 1.53 respectively). The association was not significant for glycemia. In terms of age, we found that the older the patient, the higher the risk of CKD progression.

Regarding the risk of CKD progression based on the condition's stage at the moment of diagnosis (and taking stage 4 patients as a reference point) we found that the risk of having the first progression increases as the stage at the moment of diagnosis decreases. Hence, the risk of progression for patients in stage 1 is 38.75 times higher than the risk for those in stage 4 .

The model used to analyze the need for RRT also showed that patients belonging to the RPP have $44.9 \%$ less probability of requiring renal replacement therapy. Similarly, attending more than 8 medical appointments means $66.2 \%$ less probability of requiring RRT.

Likewise, it was found that patients with out-of-range levels of glycemia, hemoglobin, potassium, and albumin had a higher risk of needing RRT than those with levels within the acceptable range. Out-of-range hemoglobin posed the highest risk for needing RRT (3.64), followed by albumin (2.06). Similarly, patients with a history of dyslipidemia had a higher risk of needing RRT, but the association was not significant. Additionally, it was found that being younger is a protective factor against the risk of requiring RRT.

Regarding the CKD stage at the moment of diagnosis, it was observed that patients in stage 4 , had a 9.35 times greater risk of requiring RRT than patients in stages 1,2, and 3 . 
Table 1. Number of appointments for CKD patients in the RPP. Appointments are arranged by appointment type and treating professional. Medellín 2004-2008.

\begin{tabular}{|c|c|c|c|c|}
\hline \multirow{2}{*}{ Clinical Indicators } & \multicolumn{2}{|c|}{ RPP } & \multicolumn{2}{|c|}{ CT } \\
\hline & $\mathrm{N}(\%)$ & Md (Q1 - Q3) & $\mathbf{N}(\%)$ & Md (Q1 - Q3) \\
\hline Educational appointments & $450(10.7)$ & $4(2-8)$ & $55(3.8)$ & $1(1-1)$ \\
\hline Nutritional appointments & $1630(38.8)$ & $2(1-3)$ & $320(21.9)$ & $1(1-2)$ \\
\hline Ophthalmological appointments & $226(5.4)$ & $1(1-2)$ & $184(12.6)$ & $1(1-1)$ \\
\hline Psychological appointments & $10(0.2)$ & $1(1-6)$ & $22(1.5)$ & $1(1-3)$ \\
\hline General practitioner & $2401(57.1)$ & $3(1-5)$ & $1022(70.0)$ & $3(1-5)$ \\
\hline Internist & $3909(93.0)$ & $4(2-6)$ & $773(52.9)$ & $3(1-5)$ \\
\hline Nephrologist & $1925(45.8)$ & $3(1-5)$ & $4(0.3)$ & $3.5(1.3-6.5)$ \\
\hline Total appointments & $4186(99.6)$ & $8(4-15)$ & $1316(90.1)$ & $8(4-13)$ \\
\hline
\end{tabular}

Table 2. Percentage distribution and probability of belonging to each CKD stage among patients benefiting from two types of intervention. Medellín, 2004-2008.

\begin{tabular}{|c|c|c|c|c|c|c|}
\hline \multirow{2}{*}{$\begin{array}{l}\text { Stage of the condition } \\
\text { upon diagnosis }\end{array}$} & \multicolumn{2}{|c|}{ RPP } & \multicolumn{2}{|c|}{ CT } & \multirow{2}{*}{ OR } & \multirow{2}{*}{ CI 95\% } \\
\hline & \multicolumn{2}{|c|}{ n (\%) } & \multicolumn{2}{|c|}{ n (\%) } & & \\
\hline 1 & 403 & $(9.6)$ & 148 & $(10.1)$ & 1.00 & \\
\hline 2 & 885 & $(21.1)$ & 96 & $(6.6)$ & 0.30 & $(0.22-0.4)$ \\
\hline 3 & 2354 & $(56.0)$ & 911 & $(62.4)$ & 1.05 & $(0.86-1.3)$ \\
\hline 4 & 493 & $(11.7)$ & 267 & $(18.3)$ & 1.47 & $(1.17-1.91)$ \\
\hline 5 & 67 & (1.6) & 39 & (2.7) & 1.59 & $(1-2.51)$ \\
\hline \multicolumn{7}{|c|}{$P$ value of the Chi-square test for tendency $<0.001$} \\
\hline
\end{tabular}

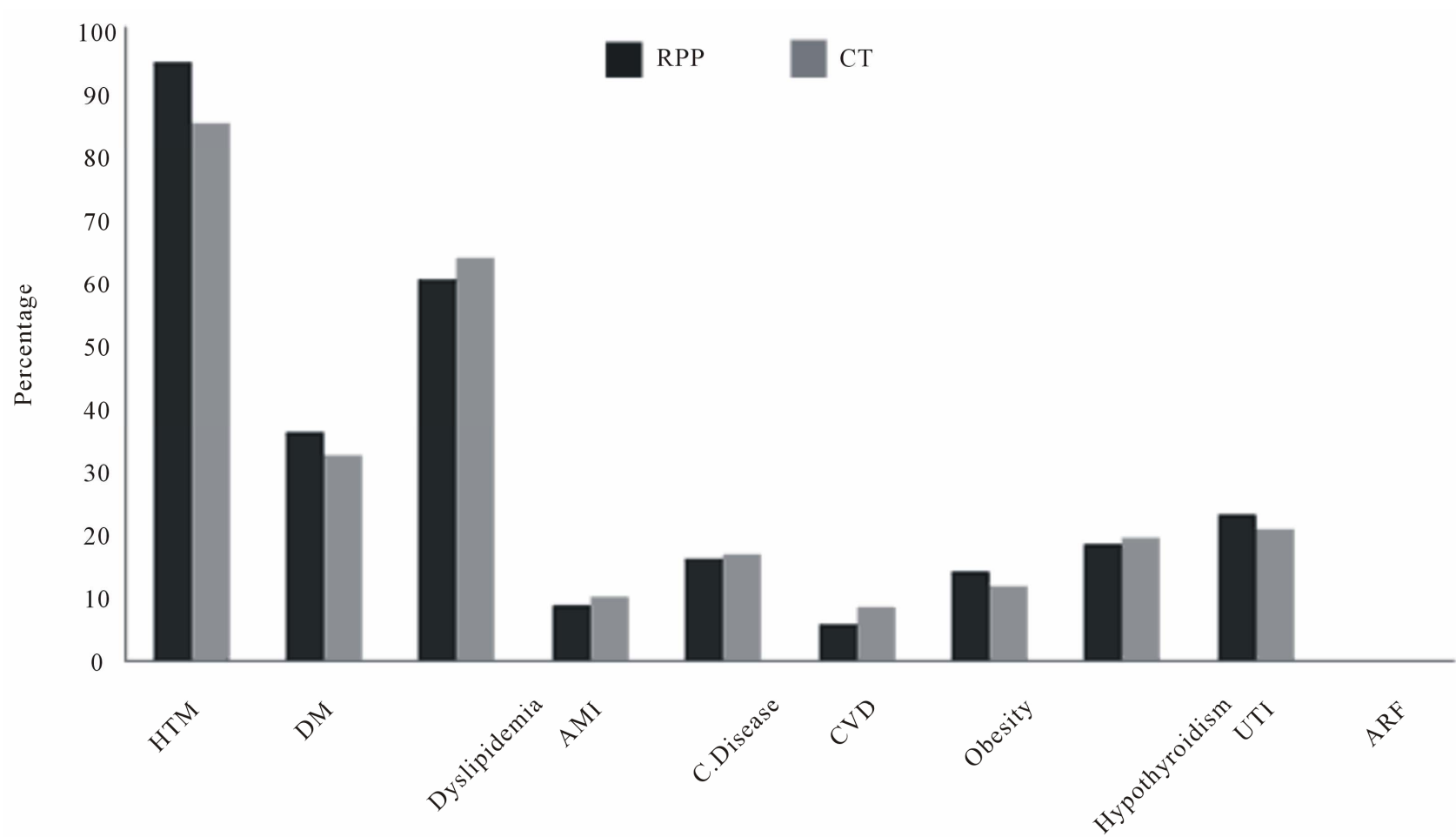

Figure 2. Comorbidities of the groups of CKD patients following a RPP and undergoing conventional treatment TC. Medellín, 2004-2008. HTN: arterial hypertension, DM: diabetes mellitus, AMI: acute myocardial infarction, C. Disease: Coronary disease, CVD: cerebrovascular disease, UTI: urinary tract infection, ARF: acute renal failure. 
Table 3. Clinical conditions and exposure to nephrotoxic and nephroprotective drugs at the end of the treatment for the CKD patients in the renal protection program, RPP, and for those undergoing conventional treatment, CT. These variables are adjusted for the basal conditions (Propensity Score). Medellín, 2004-2008.

\begin{tabular}{|c|c|c|c|c|c|c|c|c|c|}
\hline & \multirow{2}{*}{ Clinical Markers } & \multirow{2}{*}{ B } & \multirow{2}{*}{ S.E. } & \multirow{2}{*}{ Wald } & \multirow{2}{*}{ gl } & \multirow{2}{*}{$P$ value } & \multirow{2}{*}{ OR } & \multicolumn{2}{|c|}{ CI $95.0 \%$} \\
\hline & & & & & & & & IL & SL \\
\hline \multicolumn{10}{|c|}{ SAP } \\
\hline & Intervention through $\mathrm{CT}$ & 0.180 & 0.072 & 6 & 1 & 0.013 & 1.197 & 1.039 & 1.379 \\
\hline & Initial systolic arterial pressure outside of normal range & 1.013 & 0.063 & 259 & 1 & 0.000 & 2.754 & 2.434 & 3.116 \\
\hline & PS & -0.745 & 0.285 & 7 & 1 & 0.009 & 0.475 & 0.272 & 0.830 \\
\hline & Constant & -0.713 & 0.080 & 78 & 1 & 0.000 & 0.490 & & \\
\hline \multicolumn{10}{|c|}{ Fasting blood glucose } \\
\hline & Intervention through $\mathrm{CT}$ & 0.264 & 0.130 & 4 & 1 & 0.042 & 1.302 & 1.010 & 1.680 \\
\hline & Initial glycemia outside of normal range & 2.424 & 0.131 & 342 & 1 & 0.000 & 11.291 & 8.734 & 14.595 \\
\hline & PS & -0.965 & 0.560 & 3 & 1 & 0.085 & 0.381 & 0.127 & 1.143 \\
\hline & Constant & -1.742 & 0.151 & 134 & 1 & 0.000 & 0.175 & & \\
\hline \multicolumn{10}{|c|}{ Hbalc } \\
\hline & Intervention through $\mathrm{CT}$ & 1.322 & 0.244 & 29 & 1 & 0.000 & 3.751 & 2.327 & 6.046 \\
\hline & Initial Hba1c levels outside of normal range & 2.911 & 0.202 & 209 & 1 & 0.000 & 18.377 & 12.380 & 27.276 \\
\hline & PS & -1.650 & 0.965 & 3 & 1 & 0.087 & 0.192 & 0.029 & 1.274 \\
\hline & Constant & -1.995 & 0.282 & 50 & 1 & 0.000 & 0.136 & & \\
\hline \multicolumn{10}{|c|}{ Total cholesterol } \\
\hline & Intervention through $\mathrm{CT}$ & -0.252 & 0.116 & 5 & 1 & 0.030 & 0.778 & 0.620 & 0.975 \\
\hline & Initial total cholesterol level outside of normal range & 2.064 & 0.097 & 452 & 1 & 0.000 & 7.879 & 6.514 & 9.530 \\
\hline & PS & -0.962 & 0.437 & 5 & 1 & 0.028 & 0.382 & 0.162 & 0.901 \\
\hline & Constant & -1.473 & 0.131 & 126 & 1 & 0.000 & 0.229 & & \\
\hline \multicolumn{10}{|c|}{ Hemoglobin } \\
\hline & Intervention through $\mathrm{CT}$ & -0.228 & 0.120 & 4 & 1 & 0.056 & 0.796 & 0.630 & 1.006 \\
\hline & Initial hemoglobin level outside of normal range & 2.376 & 0.151 & 248 & 1 & 0.000 & 10.763 & 8.005 & 14.470 \\
\hline & PS & 1.450 & 0.466 & 10 & 1 & 0.002 & 4.262 & 1.710 & 10.619 \\
\hline & Constant & -1.391 & 0.127 & 120 & 1 & 0.000 & 0.249 & & \\
\hline \multicolumn{10}{|c|}{ Albumin } \\
\hline & Intervention through $\mathrm{CT}$ & 0.694 & 0.460 & 2 & 1 & 0.131 & 2.001 & 0.813 & 4.930 \\
\hline & Initial albumin level outside of normal range & 2.925 & 0.220 & 176 & 1 & 0.000 & 18.639 & 12.101 & 28.708 \\
\hline & PS & -0.299 & 1.094 & 0 & 1 & 0.785 & 0.742 & 0.087 & 6.333 \\
\hline & Constant & -2.960 & 0.293 & 102 & 1 & 0.000 & 0.052 & & \\
\hline \multicolumn{10}{|c|}{ Calcium } \\
\hline & Intervention through $\mathrm{CT}$ & -0.111 & 0.248 & 0 & 1 & 0.656 & 0.895 & 0.551 & 1.455 \\
\hline & Initial calcium level outside of normal range & 1.827 & 0.177 & 107 & 1 & 0.000 & 6.218 & 4.399 & 8.788 \\
\hline & PS & 1.258 & 0.715 & 3 & 1 & 0.078 & 3.519 & 0.867 & 14.285 \\
\hline & Constant & -1.985 & 0.203 & 95 & 1 & 0.000 & 0.137 & & \\
\hline \multicolumn{10}{|c|}{ Potassium } \\
\hline & Intervention through $\mathrm{CT}$ & 0.391 & 0.161 & 6 & 1 & 0.015 & 1.479 & 1.078 & 2.029 \\
\hline & Initial potassium level outside of normal range & 1.523 & 0.169 & 81 & 1 & 0.000 & 4.586 & 3.295 & 6.384 \\
\hline & PS & 0.268 & 0.548 & 0 & 1 & 0.625 & 1.307 & 0.447 & 3.825 \\
\hline & Constant & -1.642 & 0.147 & 124 & 1 & 0.000 & 0.194 & & \\
\hline \multicolumn{10}{|c|}{ Nephrotoxic drugs } \\
\hline & Intervention through $\mathrm{CT}$ & 0.491 & 0.123 & 16 & 1 & 0.000 & 1.633 & 1.283 & 2.080 \\
\hline & Initial nephrotoxic drug consumption & 2.811 & 0.118 & 564 & 1 & 0.000 & 16.624 & 13.182 & 20.966 \\
\hline & PS & 0.590 & 0.485 & 1 & 1 & 0.224 & 1.803 & 0.697 & 4.668 \\
\hline & Constant & -3.426 & 0.144 & 567 & 1 & 0.000 & 0.033 & & \\
\hline
\end{tabular}


Continued

\begin{tabular}{|c|c|c|c|c|c|c|c|c|}
\hline \\
\hline \multicolumn{9}{|l|}{$\begin{array}{l}\text { Nephroprotective drugs } \\
\text { Intervention through CT }\end{array}$} \\
\hline \multicolumn{9}{|c|}{$\begin{array}{lllllll}0.102 & 674 & 1 & 0.000 & 14.045 & 11.505 & 17.147\end{array}$} \\
\hline \multicolumn{9}{|c|}{$0.000 \quad 28.935 \quad 14.325 \quad 58.44$} \\
\hline Constant & -4.263 & 0.131 & 1.059 & 1 & 0.000 & 0.014 & & \\
\hline
\end{tabular}

Table 4. Logistic regression models for the variables "first progression" and "need for renal replacement therapy" amongst the CKD patients in the RPP and those following conventional treatment (CT), 2004-2008.

\begin{tabular}{|c|c|c|c|c|c|c|c|c|}
\hline \multirow{3}{*}{ Variables in the model } & \multicolumn{4}{|c|}{ First progression ${ }^{1}$} & \multicolumn{4}{|c|}{${\text { Need for } \text { RRT }^{2}}^{2}$} \\
\hline & \multirow{2}{*}{$P$ value } & \multirow{2}{*}{ OR } & \multicolumn{2}{|c|}{ CI 95\% } & \multirow{2}{*}{$P$ value } & \multirow{2}{*}{ OR } & \multicolumn{2}{|c|}{ CI $95 \%$} \\
\hline & & & IL & SL & & & IL & SL \\
\hline Treatment with RPP* & 0.000 & 0.483 & 0.336 & 0.696 & 0.010 & 0.551 & 0.345 & 0.882 \\
\hline Attending more than 8 appointments & 0.000 & 0.287 & 0.219 & 0.376 & 0.000 & 0.338 & 0.208 & 0.550 \\
\hline Age & 0.000 & 1.032 & 1.023 & 1.041 & 0.020 & 0.984 & 0.972 & 0.997 \\
\hline History of dyslipidemia & 0.010 & 1.354 & 1.078 & 1.701 & 0.250 & 1.243 & 0.857 & 1.801 \\
\hline \multicolumn{9}{|l|}{ Stage of the condition upon diagnosis } \\
\hline Stage 4 & 0.000 & & 1 (Referen & & 0.000 & 10.354 & 7.02 & 15.273 \\
\hline Stage 3 & 0.000 & 2.018 & 1.468 & 2.775 & & & & \\
\hline Stage 2 & 0.000 & 10.500 & 6.857 & 16.090 & & \multicolumn{3}{|c|}{1 (Reference) $^{* *}$} \\
\hline Stage 1 & 0.000 & 39.750 & 22.996 & 68.707 & & & & \\
\hline \multicolumn{9}{|l|}{ Clinical Indicators } \\
\hline Glycemia outside of normal range & 0.870 & 1.025 & 0.757 & 1.389 & 0.010 & 1.899 & 1.212 & 2.977 \\
\hline Hemoglobin level outside of normal range & 0.000 & 2.917 & 2.307 & 3.689 & 0.000 & 3.647 & 2.531 & 5.256 \\
\hline Potassium level outside of normal range & 0.010 & 1.458 & 1.116 & 1.903 & 0.020 & 1.616 & 1.098 & 2.380 \\
\hline Albumin level outside of normal range & 0.010 & 1.535 & 1.096 & 2.148 & 0.000 & 2.060 & 1.314 & 3.231 \\
\hline Constant & 0.000 & 0.026 & & & 0.000 & 0.088 & & \\
\hline
\end{tabular}

1. First progression: ${ }^{2}$ Nagelkerke $\mathrm{R}=27.55 \% ; 2$. Need for RRT: ${ }^{2}$ Nagelkerke $\mathrm{R}=32.8 \%$; ${ }^{*}$ Reference intervention; ${ }^{* *}$ Stages 1,2 , and 3 were grouped as a reference in order to compare them with stage 4 in model 2.

\section{Discussion}

The Colombian healthcare system now considers CKD as a catastrophic and very costly pathology. Thanks to this, patients now have more and better access to the services designed to address it. This has also helped health insurance companies and other institutions reduce the impact of the costs associated with this condition. Consequently, improvements in users' health are expected, as well as a better use of the health system's resources [8].

However, a great deal of studies have focused on the final stages of CKD, both in the world and in Colombia, and it is considered that more evidence should be generated regarding the effectiveness of the programs used before RRT. In this sense, this study demonstrated the effect of a predialysis intervention program complying with strategies for generating scientific evidence $[8,20]$, such as early patient uptake, comprehensive care, and treatment of comorbidities.
The fact that patients are started on the renal protection program when they are at the initial states of their condition (which is demonstrated by the fact that $30 \%$ of the patients join the program at stages 1 and 2 in comparison with CT patients) demonstrates the significant effect of this program's active search for patients, something which should be included in all secondary prevention strategies.

The results show that the RPP patients receive more interdisciplinary care. This is evidenced by the proportion of patients seen by doctors and by the higher number of medical appointments involving different health professionals. This is compliant with the interdisciplinarity proposed by a number of studies, which show differences in CKD outcomes when comorbidities are treated by multiple disciplines [21-23].

The higher percentage of educational appointments and the comprehensive care provided by the RPP show how it can be used for prevention. One of its positive 
effects could be a lower exposure to nephrotoxic drugs and higher rates of nephroprotective drugs usage.

The high number of general medical appointments for patients undergoing CT may be due to the particular characteristics of the on-demand type of health care provided by this kind of intervention. Additionally, the greater quantity of ophthalmology appointments observed in patients following CT may signify that this target organ is more affected in their case. This could be linked to the fact that their arterial hypertension, glycemia, or glycated hemoglobin levels were not within normal ranges at the end of their treatment.

The literature recommends that patients in stage 3 and onwards should be managed by nephrology professionals $[24,25]$. Despite this, only 4 individuals following CT had this kind of treatment, making it impossible to compare the groups studied.

In terms of clinical indicators, it's worth noting that, although the logistic regression model showed that having out-of-range hemoglobin and albumin levels represented a high risk of having the first CKD progression and needing RRT, no significant differences were found between the RPP and CT patients regarding the management of these markers upon adjusting for the basal levels and PS. This shows how necessary it is to implement or improve the actions aiming at keeping the levels of these indicators within normal ranges. This would greatly contribute to reducing the risk of renal function impairment [26].

Likewise, it is also worth mentioning that the RPP shows shortcomings regarding the management of total cholesterol when compared to CT-it is therefore necessary to identify the cause of such shortcomings, since inappropriate management of this indicator (i.e. high cholesterol values) is a risk factor for renal function impairment. Moreover, high cholesterol is also a risk factor for cardiovascular disease, which in turn is the main cause of morbidity and mortality among CKD patients [27].

Patients diagnosed with stage 4 CKD had a high risk of needing RRT compared with those diagnosed at earlier stages of their condition. This demonstrates the importance of early detection of CKD in order to prevent or slow down the deterioration of renal function and the resulting deterioration of patients' quality of life, 1-2 as well as the economic consequences for the various elements of the General System for Health-Related Social Security $[28,29]$.

Although the results obtained in this study in most cases showed differences favoring the RPP, it can also be observed that the proportion of patients attending appointments offered by the program and having clinical markers within the normal ranges could be improved. However, the findings regarding the protection levels provided by the RPP against the studied outcomes exceed $50 \%$ and explain more than $25 \%$ of its variability, thus suggesting that it is possible to have more effectiveness and a higher effect on the delay of CKD progression.

Implementing strategies for early intervention for CKD patients is an effective contribution to help keep the clinical markers associated with renal damage within normal ranges. It also has a positive effect on the treatment of the condition. Therefore, encouraging the implementation of such strategies optimizes the use of resources and improves patient prognosis.

\section{Acknowledgements}

The authors would like to thank Colciencias, the University of Antioquia, the EPS Sura health insurance company, and the insurance company offering CT. Study sponsor not had any role in study design; collection, analysis, and interpretation of data; writing the report; and the decision to submit the report for publication. We, the authors, declare that there were no conflicts of interest during this study.

\section{REFERENCES}

[1] R. C. Atkins, "The Epidemiology of Chronic Kidney Disease," Kidney International Supplement, Vol. 94, 2005, pp. S14-18. doi:10.1111/j.1523-1755.2005.09403.x

[2] R. C. Atkins, "The Changing Patterns of Chronic Kidney Disease: The Need to Develop Strategies for Prevention Relevant to Different Regions and Countries," Kidney International Supplement, Vol. 98, 2005, pp. S83-85. doi:10.1111/j.1523-1755.2005.09815.x

[3] R. S. Barsoum, "Chronic Kidney Disease in the Developing World," The New England Journal of Medicine, Vol. 354, No. 10, 2006, pp. 997-999. doi:10.1056/NEJMp058318

[4] S. L. White, A. Cass, R. C. Atkins and S. J. Chadban, "Chronic Kidney Disease in the General Population," Advances in Chronic Kidney Disease, Vol. 12, No. 1, 2005, pp. 513. doi:10.1053/j.ackd.2004.10.009

[5] N. Lameire, K. Jager, W. Van Biesen, D. de Bacquer and R. Vanholder, "Chronic Kidney Disease: A European Perspective," Kidney International Supplement, Vol. 99, 2005, pp. S30-38. doi:10.1111/j.1523-1755.2005.09907.x

[6] Cuenta de Alto Costo, "Contenidos Mínimos Indispensables Para la Gestión Del Riesgo Renal en un Programa de Atención a Pacientes Adultos con Enfermedad Renal Crónica, Sin Terapia de Reemplazo Renal en Colombia, Definiciones Técnicas Basadas en Evidencias," 2011. http://www.cuentadealtocosto.org/patologias/ERC/docs/c onsenso/programas_ERC.pdf

[7] Ministerio de la Protección Social, República de Colombia, "Guía Para el Manejo de la Enfermedad Renal Crónica y Modelo de Prevención y Control de la Enfermedad Renal Crónica. Componente de un Modelo de Salud Re- 
nal," 2011.

http://pos.gov.co/Documents/Archivos/ERC.pdf

[8] F. Martínez and M. Valencia "Modelo en Prevención y Control de la Enfermedad Renal Crónica. Componente de un Modelo de Salud Renal,” Bogotá, 2005.

[9] Ministerio de Protección Social, República de Colombia, "Cuenta de Alto Costo," 2011. http://www.cuentadealtocosto.org/CAC.html

[10] B. M. Curtis, P. Ravani, F. Malberti, F. Kennett, P. A. Taylor, O. Djurdjev and A. Levin, "The Short- and LongTerm Impact of Multi-Disciplinary Clinics in Addition to Standard Nephrology Care on Patient Outcomes," Nephrology Dialysis Transplantation, Vol. 20, No. 1, 2005, pp. 147-154. doi:10.1093/ndt/gfh585

[11] C. Black, P. Sharma, G. Scotland, K. McCullough, D. McGurn, L. Robertson, N. Fluck, A. MacLeod, P. McNamee, G. Prescott and C. Smith, "Early Referral Strategies for Management of People with Markers of Renal Disease: A Systematic Review of the Evidence of Clinical Effectiveness, Cost-Effectiveness and Economic Analysis," Health Technology Assessment, Vol. 14, No. 21, 2010, pp. 1-184.

[12] R. Pellicano, P. G. Kerr and R. C. Atkins, "What Have We Learned from Clinical Trials on Prevention?" Kidney International Supplement, Vol. 94, 2005, pp. 101-106. doi:10.1111/j.1523-1755.2005.09424.x

[13] L. D. B. Gray, "Outcomes Research in Nutrition and Chronic Kidney Disease: Perspectives, Issues in Practice, and Processes for Improvement," Advances in Chronic Kidney Disease, Vol. 12, No. 1, 2005, pp. 96-106. doi:10.1053/j.ackd.2004.10.012

[14] M. M. Slowik, "Early Education of Patients with Chronic Renal Insufficiency: The Healthy Start program. Case Study of the Anemic Patient," Nephrology Nursing Journal, Vol. 28, No. 6, 2001, pp. 643-646.

[15] K. Tungsanga, C. Ratanakul, W. Pooltavee, N. Mahatanan, A. I. Na Ayuthaya and S. Rodpai, "Experience with Prevention Programs in Thailand," Kidney International Supplement, Vol. 94, 2005, pp. 68-69. doi:10.1111/j.1523-1755.2005.09417.x

[16] R. T. Gansevoort, Van der Heij, C. A. Stegeman, F. T. De Charro, M. G. Nieuwenhuizen and D. De Zeeuw, "Trends in the Incidence of Treated End-Stage Renal Failure in the Netherlands: Hope for the Future?" Kidney International Supplement, Vol. 92, 2004, pp. 7-10. doi:10.1111/j.1523-1755.2004.09202.x

[17] National Kidney Foundation, "K/DOQI Clinical Practice Guidelines for Chronic Kidney Disease: Evaluation, Classification, and Stratification," Recommendations for clinical Performance Measures, 2007. http://www.kidney.org/professionals/kdoqi/guidelines_ck d/p8_cpm.htm

[18] M. Katz, "Multivariable Analysis. A Practical Guide for Clinicians," 2nd Edition, Cambridge, 2006.

[19] A. Ruiz and L. Morillo, "Epidemiología Clínica. Investigación Clínica Aplicada," Editorial Panamericana, Bogotá, 2004.

[20] H. Tazeen, "The Growing Burden of Chronic Kidney Disease in Pakistan," The New England Journal of Medicine, Vol. 9, No. 10, 2006, pp. 995-997.

[21] B. J. Barrett, "Applying Multiple Interventions in Chronic Kidney Disease," Seminars in Dialysis, Vol. 16, No. 2, 2003, pp. $157-164$. doi:10.1046/j.1525-139X.2003.16032.x

[22] D. C. Mendelssohn, "Coping with the CKD Epidemic: The Promise of Multidisciplinary Team-Based Care," Nephrology Dialysis Transplantation, Vol. 20, No. 1, 2005, pp. 10-12. doi:10.1093/ndt/gfh636

[23] R. Pellicano, R. C. Atkins and P. G. Kerr, "What Have We Learned from Clinical Trials on Prevention?" Kidney International Supplement, Vol. 94, 2005, pp. 101-106. doi:10.1111/j.1523-1755.2005.09424.x

[24] B. J. Pereira, "Overcoming Barriers to the Early Detection and Treatment of Chronic Kidney Disease and Improving Outcomes for End-Stage Renal Disease," American Journal of Managed Care, Vol. 8, No. 4, 2002, pp. S122-S135.

[25] "Chronic Kidney Disease: Stating the Managed Care Case for Early Treatment Discussion and Consensus of Presentations of Economic Analyses, Managed Care Organization Case Studies, and Opportunities for Intervention in a Managed Care Setting," American Journal of Managed Care, Vol. 8, No. 4, 2002, pp. S114-S120.

[26] A. Levin, "The Need for Optimal and Coordinated Management of CKD," Kidney International, Vol. 68, No. 99, 2005, pp. S7-S10.

[27] W. F. Owen, "Patterns of Care for Patients with Chronic Kidney Disease in the United States: Dying for Improvement," Journal of the American Society of Nephrology, Vol. 14, No. 2, 2003, pp. 76-80. doi:10.1097/01.ASN.0000070145.00225.EC

[28] Grupo de Economía de la Salud, "Evaluación Económica en Salud: Tópicos Teóricos y Aplicaciones en Colombia," Observatorio de la Seguridad Social, 2006.

[29] B. Manns, B. Hemmelgan, M. Tonelli, Au Flora, T. C. Chiasson, J. Dong and S. Klarenbach, "Population Based Screening for Chronic Kidney Disease: Cost Effectiveness Study," BMJ, Vol. 341, 2010, p. 5869. 